\title{
Is the bioanalytical community ready to revise the use of certain validation concepts and nomenclature?
}

\author{
Having a harmonized definition of what defines a method and with the differentiation of the validation \\ experiments, we believe it is possible to improve clarity in the regulatory requirements..."
}

Keywords: between-batch variation $\approx$ method characterization $\approx$ method qualification $\approx$ method validation $\approx$ overall precision upper limit of calibration $=$ validation samples

For more than 10 years the US FDA Guidance on Bioanalytical Method Validation [101] has provided a common foundation for bioanalytical scientists. The general validation process along with concepts and nomenclature has been broadly adopted by the bioanalytical community even though specific scientific issues have been, and are still debated. However, in light of the recently published European Medicines Agency (EMA) Guideline on Bioanalytical Method Validation [102] and the ongoing process of publishing a revised FDA guidance, we believe it is time to reconsider some commonly used and accepted expressions that are creating confusion. In the following we would like to discuss the concepts of full validation, qualified methods, ULOQ, QCs, and between-batch accuracy and precision.

\section{What is a full validation \& what defines a validated method?}

According to both the EMA and FDA a full validation, somewhat cryptically, is "Establishment of all validation parameters to apply to sample analysis for the bioanalytical method for each analyte" [101,102]. Furthermore, EMA states that "A full method validation should be performed for any analytical method whether new or based upon literature" [102]. A common interpretation of these guidelines is that a full method validation requires all the experiments given in the regulatory documents to be performed using the method under validation, and omission of any experiments is by definition a partial validation. Instead of focusing on a full validation, that is, the range of experiments to be performed, we recommend switching focus onto the result - the fully validated method for which all the validation parameters requested by the regulatory guidelines have been assessed. By doing so, we think that conflicting messages such as the one presented in the EMA guidelines regarding full or partial validation of additional species [102] (e.g., as highlighted by [1]) can be avoided.

By changing the focus to the validated method, there is a need to define the bioanalytical method. As a starting point for the discussion, we advocate that a validated method consist of a method procedure supplemented with data from validation experiments. The method procedure for an analyte/analytes can be defined by: the calibration range, the matrix of the calibration standards, the sample-processing technique (e.g., protein precipitation), and the separation and detection technique (e.g., LC-MS/MS, but not a specific instrument or LC column). Changes to any of these defining parameters will result in a new method procedure that will need to be fully validated. The expression 'matrix of the calibration standards' allows for the possibility of using a different calibration matrix (e.g., human plasma) for the analysis of rare matrices [102] or, for example, human plasma as a matrix for the calibration samples in the analysis of plasma samples of other species (e.g., rat and dog) [2]. In both cases, the use is justified with validation samples prepared in matrix from the test species. Furthermore, following the reasoning above, we suggest that the concept partial validation should be restricted to a response to a method change (a change that is not affecting any of the defining parameters) or an extension (e.g., additional long-term stability data) of a validated method. Thus, a partial validation can never be a standalone exercise.

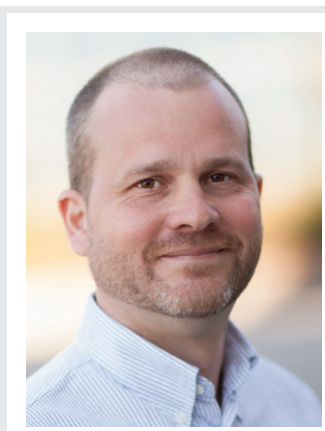

\section{Anders $\mathrm{O}$ Olsson}

Author for correspondence: Regulatory Bioanalysis, AstraZeneca R\&D Möndal, SE-43I 89 Mölndal, Sweden Tel.: +4631 7762863 Email: anders.o.olsson@ astrazeneca.com

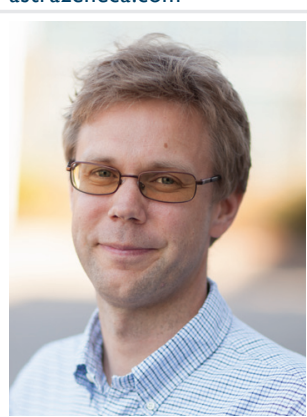

Mathias Liljeblad

Regulatory Bioanalysis, AstraZeneca R\&D Möndal, SE-43I 89 Mölndal, Sweden

Authors continued overleaf 


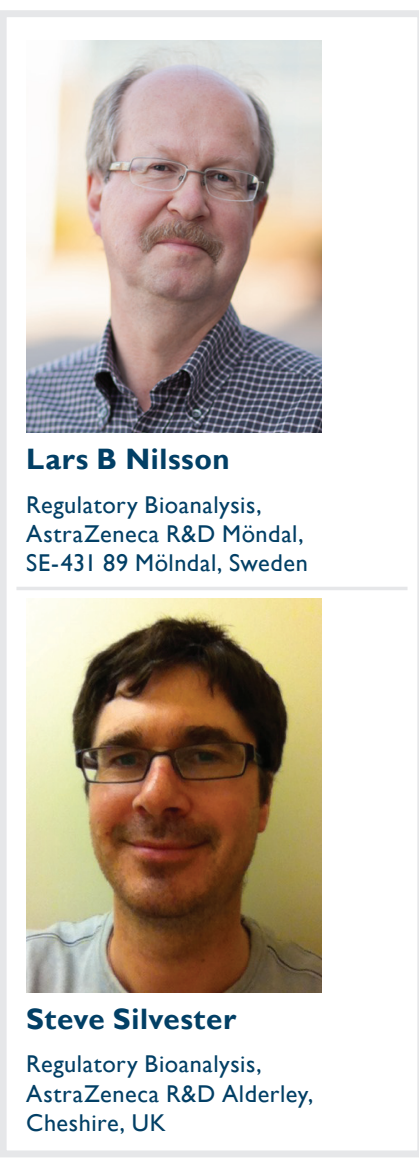

gulatory Bioanalysis, AstraZeneca R\&D Alderley, Cheshire, UK
To guide the decision regarding which parameters need to be reassessed in response to changes to the method, it is useful to differentiate among the validation experiments. These can be divided into two categories: first, method-specific experiments, designed to show the performance and quality of the method procedure; second, stability experiments to show that the integrity of the analyte/analytes is maintained between sampling and analysis. The method-specific experiments are both method and matrix specific and include both within- and between-batch accuracy and precision, selectivity, matrix effects, effect of dilution, integrity of processed samples and incurred sample reanalysis. These experiments must consequently be performed for each new method procedure. The stability experiments are dependent on the matrix and cover assessment of freeze-thaw, short- and long-term stability, stability in stock solution, stability in blood and stability in incurred samples. Since these experiments are not dependent on the bioanalytical method, there is the potential and sound scientific rational to reference previously generated results when applicable. However, although the stability itself is not method dependent, there might be situations where the method affects the results of the stability experiments. Such a case is if a degradation of the matrix gives rise to a changed matrix effect in combination with the use of an internal standard that does not compensate for the change. This kind of special case is beyond the scope of this general discussion, but should always be considered when developing new methods. In summary, a new fully validated method will include the results from the method-specific experiments, supplemented by necessary stability data, new or previously established when available.

Having a harmonized definition of what defines a method and with the differentiation of the validation experiments, we believe it is possible to improve clarity in the regulatory requirements regarding the type of experiments that must be performed to achieve a fully validated method. It would further create the possibility to harmonize the experimental requirements for partial validations in respect to certain changes in a method. However, we fully realize the challenges of attaining harmonization on what defines a method and, until there is a consensus, we believe it is beneficial for each company/institution to describe their method defining parameters in a standard operating procedure.
Qualified or characterized methods?

At the 2006 Crystal City III meeting in Arlington (VA, USA) and in the following conference proceedings it was recognized that the validation requirements for methods used for the quantification of metabolites in a MIST context should proceed using a flexible, 'tiered' approach [3]. In an attempt to bring further clarity to that statement, the European Bioanalysis Forum (EBF) has proposed to categorize methods in three quality levels: screening, qualified and validated methods [4]. The EBF has also stated that the concept of qualified method can be used in other type of studies, such as preclinical pharmacokinetic and/or pharmacodynamic studies, mechanistic toxicokinetic studies or other studies where the application of a fully validated method is not needed or not possible. The term 'qualified method' has, hence, been used as a generic label of methods that are not fully validated according to regulatory guidelines. In practice, the qualification of a method is often a routine process consisting of, for example, a selection of the experiments given in the guidance documents, sometimes with wider acceptance criteria. Thus, a method labeled 'qualified' after a routine qualification exercise is not automatically fit for its intended use, since the actual method requirements in the study of interest can vary quite substantially. In this respect, the label 'qualified' is misleading.

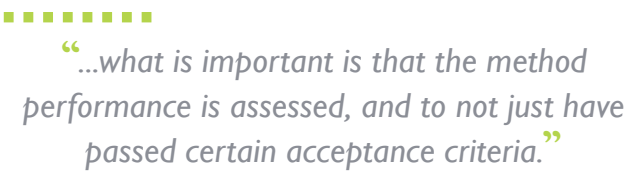

We believe that for methods used in the studies mentioned above, what is important is that the method performance is assessed, and to not just have passed certain acceptance criteria. Such an assessment should be able to characterize the method performance with a reasonable degree of certainty at a reasonable cost. Before commencing a study, or when evaluating the study results, the performance of the method should be evaluated against the requirements of the study. By referring to the assessment of the method performance as a characterization instead of a qualification we think that a change in mindset can be achieved: from a 'tick-in-the-box' approach to one based upon scientific reasoning. A continued discussion within the bioanalytical community on best practice regarding characterization of methods will hopefully result in more accurate conclusions and optimal use of resources. 


\section{Upper limit of quantification}

In regulatory bioanalysis, it is a well-established practice that sample concentrations should only be reported when the analyte response is within the limits of the nominal concentrations of the calibration standards. Therefore, during most validation studies the possibility of diluting samples into the calibration range is demonstrated in order to justify determination of analyte concentrations higher than the highest calibration standard.

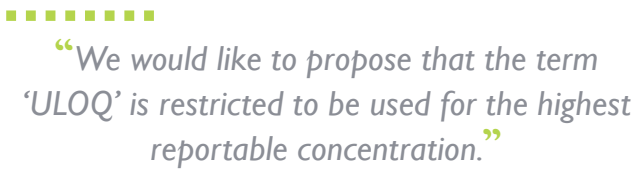

It is common practice to use the abbreviation ULOQ for the concentration of the highest calibration standard in the calibration curve. This is probably in part a result of the FDA statement, "The highest standard will define the upper limit of quantification (ULOQ) of an analytical method" [101]. However, by using the abbreviation ULOQ to define the concentration of the highest calibration standard, many safety/clinical studies will report concentrations that are higher than the upper limit of quantification, which is an obvious contradiction. We would like to propose that the term 'ULOQ' is restricted to be used for the highest reportable concentration. If we disregard the fact that both regulatory agencies switch between the terms 'amount' and 'concentration', this is in line with both the FDA definition ("The upper limit of quantification of an individual analytical procedure is the highest amount of analyte in a sample which can be quantitatively determined with pre-defined precision and accuracy") [101] and the EMA definition ("The highest amount of an analyte in a sample that can be quantitatively determined with precision and accuracy") [102]. However, especially during method validation, the concentration of the highest calibration standard is an important parameter in order to define the calibration range. Therefore, we suggest introducing the term 'upper limit of calibration' (ULOC) to define the concentration of the highest calibration standard. The ULOQ will then in practice be defined by the ULOC multiplied with the validated dilution factor.

\section{Naming of samples that are part of a validation study}

In both the FDA and the EMA documents $[101,102]$, the samples used to perform validation experiments are called QCs. However, since this term is also used as abbreviation for samples used for QC samples in routine batch analysis this can lead to misunderstandings, for instance when discussing the differentiation between the QCs for stability assessment and the QCs for batch control in long-term stability experiments.

Whilst it is acknowledged that the batch QC samples are likely to be at the same concentration as some of the validation samples, and may be used for validation purposes, we do not believe that this merits naming the two different types of samples the same. In an attempt to differentiate between the two sample types, we would like to propose the use of a specific term; for example, validation samples to describe samples used for evaluation of method validation parameters, and leave the QC abbreviation exclusively for the batch quality control samples. Although some laboratories might have definitions in their standard operating procedures to make this type of differentiation, the bioanalytical community would benefit from a common nomenclature. This would further actualize the need to clarify if it should be considered accepted to use validation samples for the dual purpose of batch acceptance and accuracy and precision determination.

\section{Estimation of variation between analytical batches}

A common approach when evaluating accuracy and precision during the validation is to analyze 5-6 replicates for each investigated concentration in each of three batches [5-8]. In such a case a real estimation of between-batch variations normally means that an ANOVA-based approach is needed. However, due to the complicated calculations, especially if the number of observations differ between occasions (e.g., if an outlier has been excluded), a common practice has emerged in which the between-batch statistics are calculated by just including all measurements (e.g., $3 \times 6$ replicates) [1]. This type of calculation has gained further acceptance with the new EMA guideline in which the description of betweenbatch calculation can be interpreted in the same way [102]. However, this measurement does not show anything regarding a potential betweenbatch variation of either precision or accuracy, and should if anything be called a 'total' or 'overall' accuracy and precision [1]. Furthermore, there is no need, in our understanding, to place an acceptance criterion on the between-batch accuracy since it will always pass as long as the 
underlying requirement for all within-batch accuracy values to be within acceptance limits is fulfilled. Thus, we would like to propose that the precision calculated on all replicates is called 'overall precision' and that a 'between-batch variation' is estimated by calculating the standard deviation of the within-batch means divided by the overall mean (result expressed as \%CV). Whilst this calculation could be questioned from a strict mathematical/statistical point of view, it is straightforward to calculate and a $<15 \%$ acceptance criterion can be used that will serve to flag methods with good within-batch precision but high between-batch variation.

In summary, we would recommend the following set of accuracy and precision calculations: within-batch accuracy, within-batch precision, overall precision and between-batch variation, all with acceptance criteria according to the regulatory guidelines.

\section{Summary}

We have discussed revisions for the common use of some concepts related to method validation. We propose clarification around the concepts of full validation by discussing a change in focus to a fully validated method, the properties that define the method, and by categorizing validation experiments into either method-specific or stability. There is a further need for an open and thorough discussion within the bioanalytical community to hopefully create a consensus around the definition of a method and the defining parameters of a method procedure. We also consider the use of the terms qualified methods, ULOQ, QC samples and between-batch accuracy and precision, and propose more appropriate alternatives such as characterized method, ULOC, validation samples, overall precision and between-batch variation. We are fully aware that a change in commonly accepted nomenclature can in itself create confusion. However, we would argue that the concepts as they stand today are confusing, and we believe that in the long term the bioanalytical community will benefit by striving to replace confusion with clarity.

\section{Financial \& competing interests disclosure}

The authors have no relevant affiliations or financial involvement with any organization or entity with a financial interest in or financial conflict with the subject matter or materials discussed in the manuscript. This includes employment, consultancies, honoraria, stock ownership or options, expert testimony, grants or patents received or pending, or royalties.

No writing assistance was utilized in the production of this manuscript.

\section{References}

1 Smith G. European Medicines Agency guideline on bioanalytical method validation: what more is there to say? Bioanalysis $4(8)$, 865-868 (2012).

2 Jacobson B, Olsson A, Fakt C, Öhman D. The use of human plasma as matrix for calibration standards in pre-clinical LC-MS/ MS methods - a way to reduce animal use. J. Pharm. Biomed. Anal. 54(4), 826-829 (2011).

3 Viswanathan C, Bansal S, Booth B et al. Workshop/conference report - quantitative bioanalytical methods validation and implementation: best practices for chromatographic and ligand binding assays. AAPS J. 9(1), E30-E42 (2007).

4 Timmerman P, Kall MA, Gordon B, Laakso S, Freisleben A, Hucker R. Best practices in a tiered approach to metabolite quantification: views and recommendations of the European Bioanalysis Forum. Bioanalysis 2(7), 1185-1194 (2010).
5 Akrami A, Hsieh F, Almon V, Bruenner BA, James C, Wong P. Simultaneous determination of a p38 MAP kinase inhibitor and its amide hydrolyzed metabolite in Cynomolgus monkey plasma by LC-MS/MS, and its application to a toxicokinetic study. J. Pharm. Biomed. Anal. 55(5), 1104-1110 (2011).

6 Gong Z, Chandler K, Webster S, Kerley R, Buist S, McCort-Tipton M. Simple and rapid determination of norethindrone in human plasma by supported liquid extraction and ultra performance liquid chromatography with tandem mass spectrometry. Talanta 91, 77-82 (2012).

7 Sillén H, Cook M, Davis P. Determination of ticagrelor and two metabolites in plasma samples by liquid chromatography and mass spectrometry. J. Chromatogr.B 878(25), 2299-2306 (2010).

8 Wei D, Sullivan M, Espinosa O, Yang L. A sensitive LC-MS/MS method for the determination of free maytansinoid DM4 concentrations - method development, validation and application to the nonclinical studies of antitumor agent DM4 conjugated hu-anti-Cripto MAb B3F6 (B3F6-DM4) in rats and monkeys. Int. J.Mass Spectrom. 312, 53-60 (2012).

\section{- Websites}

101 US FDA. Guidance for Industry: Bioanalytical Method Validation. US Department of Health and Human Services, FDA, Center for Drug Evaluation and Research, Rockville, MD, USA (2001). www.fda.gov/downloads/drugs/ guidancecomplianceregulatoryinformation/ guidances/ucm070107.pdf

102 European Medicines Agency. Guideline on validation of bioanalytical methods. EMEA/ CHMP/EWP/192217/2009 (2009). www.ema.europa.eu 